\title{
Random Amplification of Polymorphic DNAAnalysis for Genetic Characterization of Two Breeds of Dogs, German Shepherd and Japanese Spitz
}

\author{
Jarina joshi ${ }^{1}$, Lumanti Manandhar ${ }^{2}$, Pratima Shrestha ${ }^{2}$ and Rani Gupta ${ }^{2}$, \\ Rojlina Manandhar ${ }^{2}$ and Smita Shrestha ${ }^{1}$ \\ ${ }^{1}$ Central Department of Biotechnology \\ Tribhuwan University, Kirtipur, Kathmandu \\ ${ }^{2}$ Department of Biotechnology \\ Kathmandu University,Dhulikhel, Kavre \\ e-mail: jarinarjoshi@yahoo.com
}

\begin{abstract}
Random amplified polymorphic DNA (RAPD) markers were used to study genetic diversity in dog samples belonging to populations of German Shepherd and Japanese Spitz. A total of twelve samples were typed using eight RAPD primers. Out of eight primers, three primers gave result in six individuals of dogs. The phylogenetic tree constructed by the neighbor joining method based on Nei. Original measures revealed highest genetic identity found in German Shepherd as 0.9444 and highest genetic distance as 1.2809 . The analysis predicts the number of polymorphic loci as 15 and the percentage of polymorphic loci as 83.3 .
\end{abstract}

Key words: RAPD, genetic distance, japanese Spitz, german Shepherd, polymorphism

\section{Introduction}

A dog mammal of the Canidae family, is a diverse group of 34 species ranging in size and proportion from wolflike canids, south American canids and red fox canids. Dogs were domesticated from wolves as recently as 15000 years ago based on recent genetic, fossil and DNA evidences. New evidence suggests that dogs were first domesticated in east Asia, probably China. Genetic research has identified that 14 ancient dog breeds, with the oldest being Chow chow, Sharpie, Akita inu are associated with China and Japan. As human migrated around the planet, a variety of dogs migrated with them due to which diverse forms of dogs were observed through hybridistion (Stepniak 2002).

Dog breeds show extreme variation in size, appearance, color and behavior. Dogs detect sounds as low as 16 to $20 \mathrm{hz}$ frequency range and above $45 \mathrm{hz}$. On the basis of their characters dogs can be categorized as war dogs, herapy dogs, guard dogs and companion dogs. To save the extraordinary breeds of dogs, study of phenotypic diversity is not enough. Genetic identification must be done for satisfactory utilization and conservation of breeds. Genetic identity study shows how much distance exists among the breeds. Distance analysis not only discriminates two breeds but also looks after the evolutionary relationships (Lynch 1991). Polymorphic DNA markers that were shown to genetically link a trait of interest could be used for individual and pedigree identification, pathogenic diagnosis and trait improvement in genetics and breeding programme (Kemp \& Taele 1992) and is highly informative without prior knowledge of sequence information (Crowhurst 1991, Bostock 1993). RAPD has been used for population analysis in detecting genetic differences in humans and in estimation breeding in cattle (Bhattacharya 2003). 
Present work studied the genetic identity and diversity of breeds within and between German Shepherd and Japanese Spiz using different RAPD markers.

\section{Methodology}

Blood samples of dogs were collected form Royal Kennel Club (Maitighar, Kathmandu, Nepal). Blood was collected from different dogs of two breeds into a heparinised blood collection tubes and were stored at $4^{\circ} \mathrm{C}$ in refrigerator. High molecular weight DNA was isolated using protocol described by Shambrook et al. (1989). Precipitated DNA was dissolved in TE buffer and stored at $4^{\circ} \mathrm{C}$. Quality and quantity of isolated genomic DNA was evaluated by agarose gel electrophoresis and spectrophotometer.

\section{RAPD-PCR optimization and analysis of DNA fingerprints}

A set of eight decamer primers synthesized from Banglore Genei (P) Ltd. India, were used for amplification. The sequence, Guanine and cytosine
(GC) contents are as represented in Table 1. PCR reactions were carried out in $0.2 \mathrm{ml}$ microfuse tubes using programmable DNA Thermocycler (MJ research,INC, USA). Each optimized PCR reaction contained 25 ìl reaction mixtures containing $30 \mathrm{ng}$ of genomic DNA, 10 ìM of the arbitrary primer, 2.5mM of dNTP , $25 \mathrm{mM}$ of $\mathrm{MgCl}_{2}, 1.25$ unit of Taq DNA polymerase and 2.5ìl of 10 x Taq DNA polymerase buffer. Amplifications were performed using Thermal cycler programmed for 35 cycles with denaturation at $94^{\circ} \mathrm{C}$ for $1 \mathrm{~min}$, annealing at $36^{\circ} \mathrm{C}$ for $1 \mathrm{~min}$ and extension at $72^{\circ} \mathrm{C}$ for $5 \mathrm{~min}$. An initial denaturation step of $2 \mathrm{~min}$ at $94^{\circ} \mathrm{C}$ and a final hold step of $10^{\circ} \mathrm{C}$ were included in the first and last cycles, respectively. The amplification products were size-fractionated in a $1.5 \%$ agarose gel containing Ethidium bromide in Tris-borate EDTA buffer and visualized under UV transillumination. These RAPD fragments were studied for the calculation of genetic distance, genetic identity, polymorphic loci and to obtain phylogenetic tree.

Table 1. Sequence and Guanosine -Cytosine contents in RAPD Primers

\begin{tabular}{lllc}
\hline Primers & Sequence 5'-3' & Length & GC (\%) \\
\hline KMS1 & CAGGCCCTTC & $10-$ mer & 70 \\
KMS2 & TGCCGAGTCG & $10-$ mer & 70 \\
KMS3 & AATCGGGCTG & $10-$ mer & 60 \\
KMS4 & GGTCCCTGAC & $10-$ mer & 70 \\
KMS5 & GGGTAACGCC & $10-$ mer & 70 \\
KMS6 & TCGTGCTGG & $10-$ er & 60 \\
KMS7 & GGTGACGCAG & $10-$ mer & 70 \\
KMS8 & CTGAGACGGA & $10-$ mer & 60 \\
\hline
\end{tabular}

\section{Statistical analysis}

Statistical analysis was then performed by collecting data from binary matrices of zeros and ones respectively by scoring bands on agarose gel as their presence or absence. Gene frequency, polymorphic loci, genetic distance and similarity of two breeds of dogs were calculated using Population Genetic Analysis (POPGENE 32 SOFTWARE) based on Nei's (1978) genetic method. Phylogenetic tree was constructed with the UPGMA (modified from NEIGHBOR procedure of PHYLIP version 3.5) method. The cluster analysis was based on the genetic distance with UPGMA (Unweighted Pair Group Method using Arithmetic mean).

\section{Results and Discussion}

The visual estimation of DNA in agarose gel electrophoresis revealed good concentration of DNA with sharp high molecular weight bands. The OD 260 $\mathrm{nm}$ : OD $280 \mathrm{~nm}$ ratio ranged between 1.7 to 2.1 indicating a good quality of DNA.
Analysis of RAPD fingerprints
Eight primers (KMS1, KMS2, KMS3, KMS4, KMS5, KMS6, KMS7and KMS8) were chosen for RAPD analysis as used by Joshi (2007) for genetic diversity study of two cattle breeds, Gir and Kankrej. Out of which only three primers (KMS2, KMS5 \& KMS7) were able to show polymorphic fragments on two dog breeds as shown in fig. 1,2 and 3 respectively. 


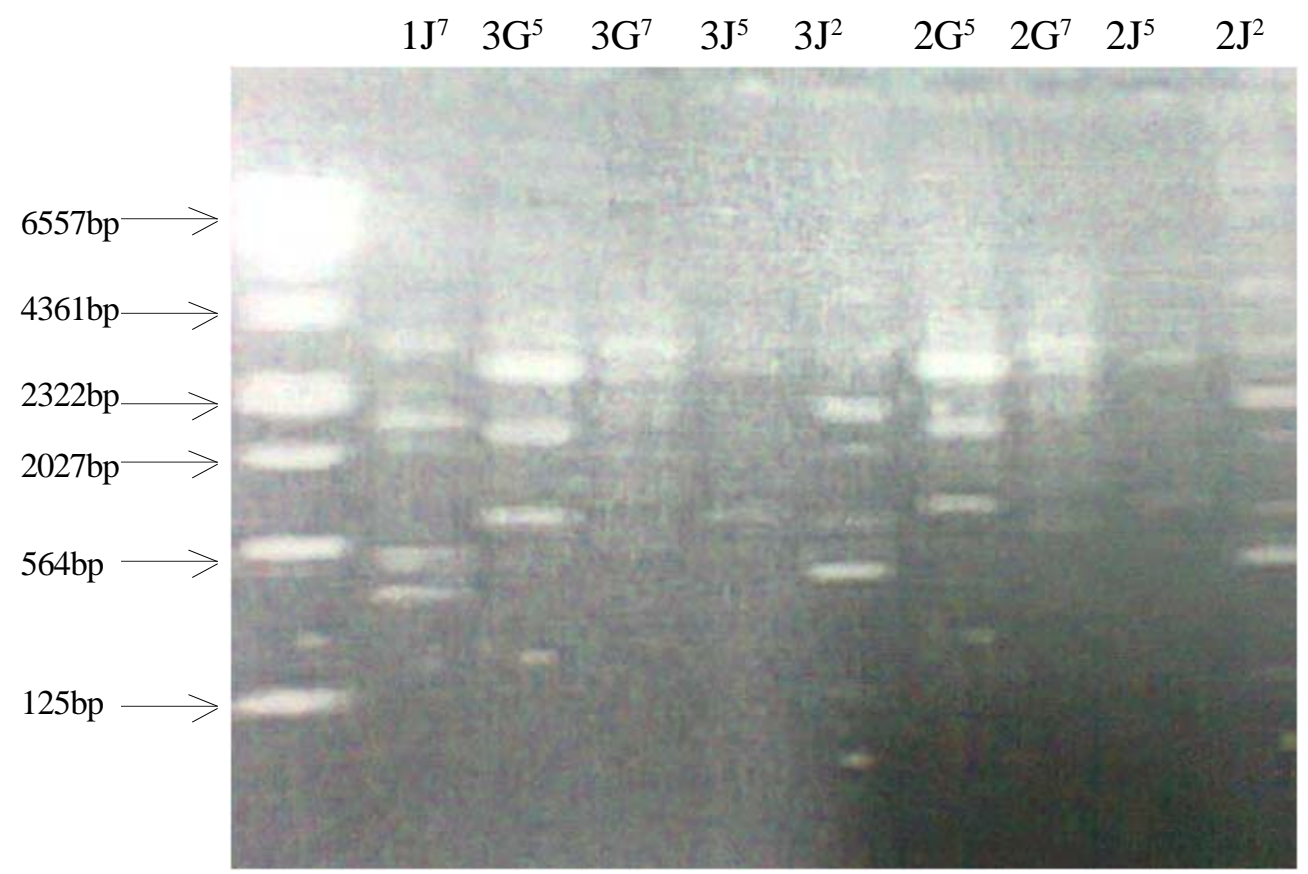

Fig.1. RAPD fingerprints of genomic DNA using primers KMS2, KMS5, KMS7

Lane 1: lambda DNA/HindIII digested marker, Lane 2: Japanese Spitz sample 1 primer KMS7, Lane 3 and 4: German Shepherd sample 3 primer KMS5 and KMS7, Lane 5 and 6 Japanese Spitz and sample 3 primers KMS5 and KMS2,Lane 7 and 8: German Shepherd sample 2 primers KMS5 andKMS7, Lane 8 and 9: Japanese Spitz sample 2 primer KMS5 and KMS2.

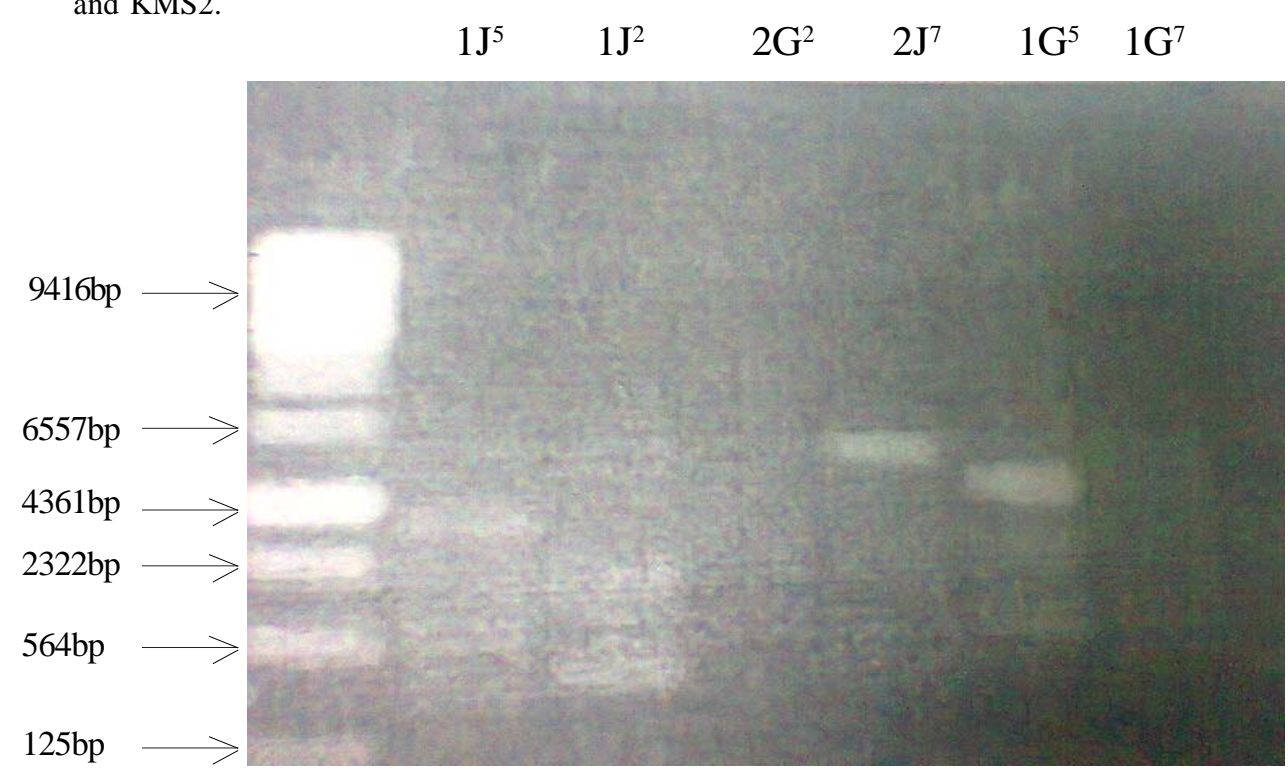

Fig. 2. RAPD fingerprints of genomic DNA using primers KMS2, KMS5, KMS7

Lane1: Lambda DNA/Hind III digested marker, Lane 2 and 3: Japanese Spitz sample1 primers KMS5 and KMS2, Lane 4: German Shepherd sample 2 primers KMS2, Lane 5: Japanese Spitz sample 2 primer KMS7, Lane 6, 7 and 8: German Shepherd sample1 primers KMS5, KMS7 and KMS2 


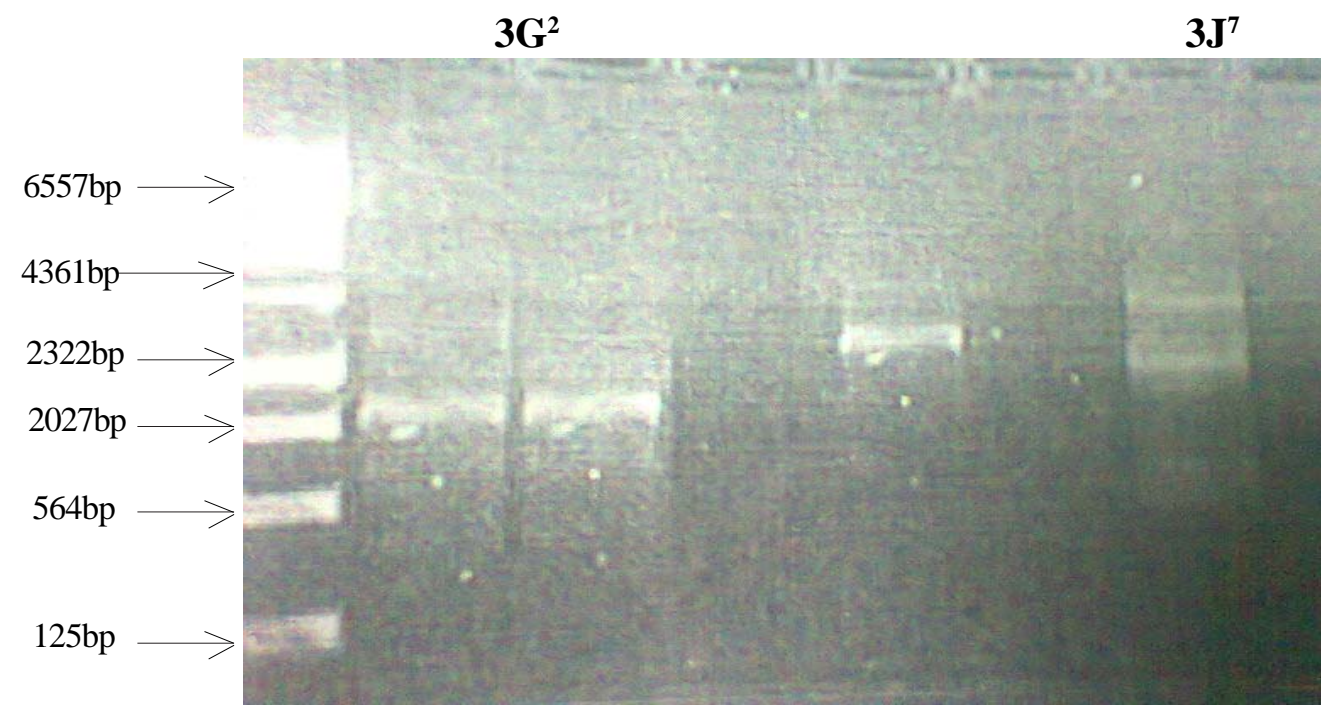

Fig. 3. RAPD fingerprints of genomic DNA using primers KMS2, KMS5 and KMS7

Lane1: lambda DNA/Hind III digested marker, Lane 3: German Shepard sample 3, Primers KMS2,

Lane 7: sample 3 Japanese Spitz KMS7

Each of these three primers yield 2 to7 RAPD fragments of the approximate size ranging from125bp to 3950. Number of polymorphic loci obtained was 15 and the percent of polymorphic loci was 83.33 . For primer KMS2, the monomorphic locus is 2100. For primer KMS5 and KMS7, the monomorphic locus is 2200 and 2250 respectively. Primer KMS2 amplified minimum fragment of band size 125bp and maximum of band size 2322bp. Similarly, primer KMS5 amplified minimum fragment of band size of $350 \mathrm{bp}$ and maximum of 3340bp. Finally, primer KMS7 amplified minimum of $400 \mathrm{bp}$ and maximum of $3950 \mathrm{bp}$ RAPD fragments. Six loci were identified in primer KMS2. From the RAPD fragments primer KMS2 produced 600bp in all the individual of German Shepherd and 2322bp in all the individuals of Japanese spitz. Primer KMS2 can be considered as a specific primer marker generating specific polymorphic band to identify the two breeds genetically. Few publication also demonstrated breed specific RAPD fingerprints (Shivakumar 1997, Anh et al. 1999). However, more study has to be adopted. Similarly, for primers KMS5 and KMS7, five and seven loci were identified respectively. Overall gene frequency for primer KMS2, KMS5 and KMS7 are listed below in Table 2, 3 and 4 respectively.

Table 2. Gene frequency for primer KMS2

\begin{tabular}{c|c|c}
\hline LOCUS & $\begin{array}{c}\text { BAND } \\
\text { SIZE(bp) }\end{array}$ & $\begin{array}{c}\text { GENE } \\
\text { FREQUENCY }\end{array}$ \\
\hline KMS2-1 & 125 & 0.2113 \\
KMS2-2 & 450 & 0.5918 \\
KMS2-3 & 600 & 0.5000 \\
KMS2-4 & 800 & 0.2113 \\
KMS2-5 & 2100 & 1.0000 \\
KMS2-6 & 2322 & 0.5000 \\
\hline
\end{tabular}

Table 3. Gene frequency for primer KMS5

\begin{tabular}{c|c|c}
\hline LOCUS & $\begin{array}{c}\text { BAND } \\
\text { SIZE(bp) }\end{array}$ & GENE FREQUENCY \\
\hline KMS5-1 & 350 & 0.1835 \\
KMS5-2 & 650 & 0.5918 \\
KMS5-3 & 1250 & 0.2113 \\
KMS5-4 & 2200 & 1.0000 \\
KMS5-5 & 3340 & 0.4226 \\
\hline
\end{tabular}

Table 4. Gene frequency for primer KMS7

\begin{tabular}{c|c|c}
\hline LOCUS & BAND SIZE & GENE FREQUENCY \\
\hline KMS7-1 & 400 & 0.2113 \\
KMS7-2 & 500 & 0.4226 \\
KMS7-3 & 650 & 0.2113 \\
KMS7-4 & 1500 & 0.2113 \\
KMS7-5 & 2250 & 1.0000 \\
KMS7-6 & 3200 & 0.7113 \\
KMS7-7 & 3950 & 0.5000 \\
\hline
\end{tabular}

Genetic distances and the genetic identities among the two dog breeds using Nei's unbiased measure of genetic identity and genetic distance assuming two populations (German Shepherd \& Japanese Spitz) gave the genetic distance of 0.4483 and genetic identity of 0.6387 between two population. Further, Nei's original measures of genetic identity and genetic distance method was used to calculate genetic distance and genetic identity of each individual of two population of dogs. The data of which are listed in Table 5. 
Jarina joshi et al./Random Amplification of

Table 5. Nei's original measures of genetic identity and genetic distance

\begin{tabular}{c|c|c|c|c|c|c}
\hline POP ID & 1 & 2 & 3 & 4 & 5 & 6 \\
\hline 1 & - & 0.5556 & 0.6667 & 0.6111 & 0.5556 & 0.5000 \\
2 & 0.5878 & - & 0.8889 & 0.3889 & 0.4444 & 0.3889 \\
3 & 0.4055 & 0.1178 & - & 0.2778 & 0.4444 & 0.3889 \\
4 & 0.4925 & 0.9945 & 1.2809 & - & 0.6111 & 0.6667 \\
5 & 0.5878 & 0.8109 & 0.8109 & 0.4925 & - & 0.9444 \\
6 & 0.6931 & 0.9445 & 0.9445 & 0.4055 & 0.0572 & - \\
\hline
\end{tabular}

From the data observed in Nei's original measures of genetic identity and genetic distance, highest genetic identity was found between German Shepherd samples 5 and $6(0.9444)$ and the least genetic identity was between Japanese Spitz sample 3 and 4 (0.2778). Similarly, highest genetic distance was observed between German Shepherd sample 4 and Japanese Spitz 3 (1.2809) and the least genetic distance was between German Shephard sample 5 and 6 (0.0572).

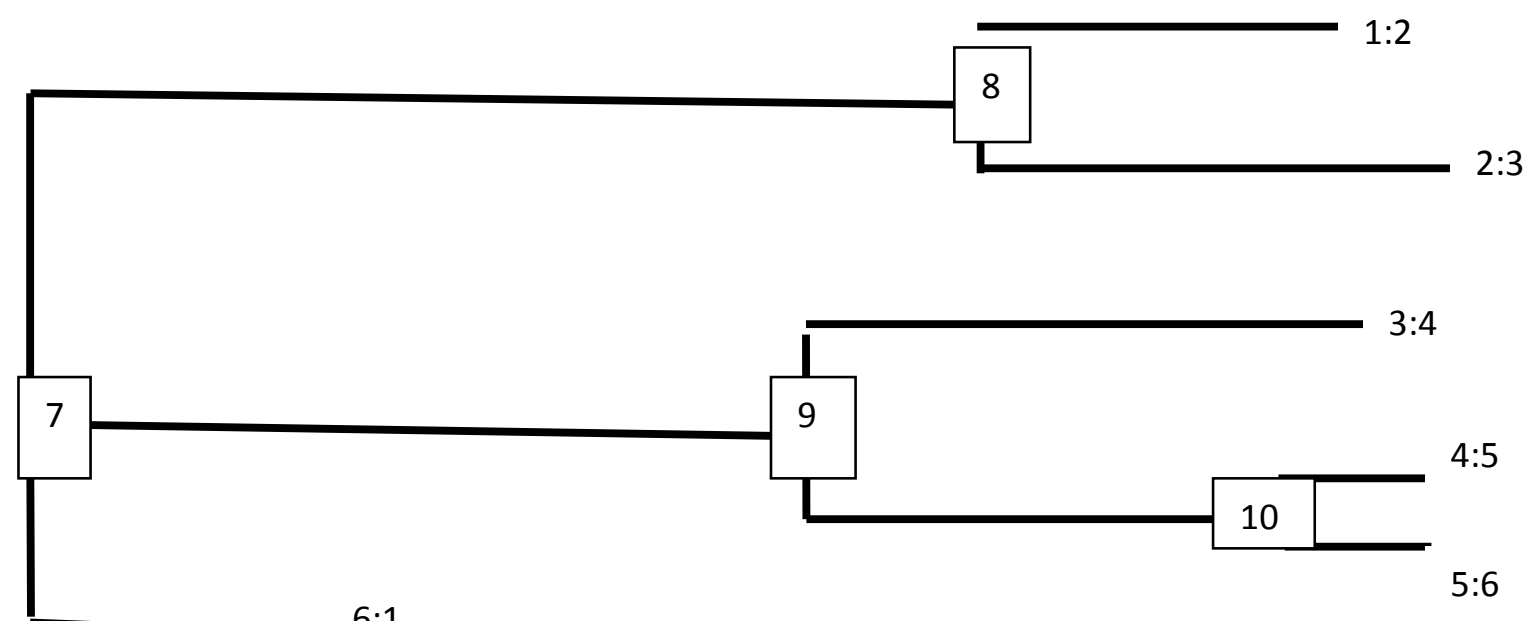

Fig. 4. Phylogenetic tree showing genetic similarity and diversity within and between two dog breeds (Nei’s 1978)

A Neighbor joining tree constructed on the basis of analysis of RAPD profiles amplified by three primers (Fig. 4) revealed that samples 2,3 (Japanese Spitz ) and sample 4,5,6 (German Shepherd) are in two different phylogenetic branches. However, the phylogenetic branch of sample 1seems to be most distinct. This suggests that sample1 of Japanese Spitz belongs to a different phylogenic linkage. This may be due to crossbreeding with individuals of other origin rather than its own origin. Meanwhile, individuals (sample 4, 5 \& 6) German Shephard shared the same phylogenetic branch which interprets that they are more identical to each other than that of individuals of Japanese Spitz which are distributed in two different phylogenetic branches (sample $2 \& 3$ in one branch and sample1in other branch). It can be inferred from the results that sample 3 of Japanese Spitz and sample 4 of German
Shepherd are separated by the largest evolutionary distance whereas samples 5 and 6 sharing the same phylogenetic branch are the most related individuals.

RAPD-PCR technique is a promising, efficient, lucid and useful molecular tool to detect genetic variability and similarity in a given population. Highest genetic identity was found between German Shepherd sample 5 and 6 (0.9444) and the highest genetic distance was found between German Shepherd sample 4 and Japanese Spitz sample 3 (1.2809). From the present study the number of polymorphic loci obtained was 15 and the percentage of polymorphic loci 83.33. This study helps in studying genetic characterization of dog genome to yield the vital information about the role of inheritance that plays in diseases such as cancer, epilepsy, heart disease and cataracts. These studies 
can enable technical efforts in comparative genomics to fight against band prevent both human and canine diseases.

\section{References}

Ahn, B.H., Y.D. Ko and Y.S. Park. 1999. Study on DNA fingerprinting in Han Woo cattle. Kor. J. Anim. Sci. 41:577-588.

Bhattacharya, T.K., P. Kumar, J.D. Joshi and S. Kumar. 2003. Estimation of inbreeding in cattle using RAPD markers. J. Dairy Res. 70:127-129.

Bostock, A., M.N. Khattak, R. Mathews and J. Burnie 1993. Comparision of PCR fingerprints by random amplification of polymorphic DNA with other molecular typing methods for Candida albicans. $J$. Gener. Microb. 139:2179-2184.

Crowhurst, R.N., B.T. Hawthrone, E. H. Rikkerink and M.D. Templeton. 1991. Differentiation of Fusarium solani, Cucurbitae races 1 and 2 by random amplification of polymorphic DNA. Curr. Genet., 20:391-396.

Joshi, J., R.K. Patel, K.M. Singh, K.J. Soni, J.B. Chauhan, D.N. Rank, C.G. Joshi and K.R.S. Sambasiva Rao. 2007.
Genome identity and diversity study in Gir and Kankrej (Bos indicus) cattle breeds using RAPD fingerprints. Biotechnology 6(3):322-327.

Kemp, S.J. and A.J. Teale.1992. Random amplified DNA polymorphism (RAPDs) and pooled DNA bovine genetic study. Anim. Genetic. 25:83-88

Lynch, M. 1991. Analysis of population genetic structure by DNA fingerprinting. In: DNA Fingerprinting Approaches and Applications. (Eds. Burke T. G., A. Dolf, J. Jeffreys \& R. Wolf), Basel, Switzerland, 113126 p.

Nei, M. 1978 Estimation of average heterozygosity and genetic distance from a small number of individuals. Genetics 89:583-890.

Shambrook, J., E.F. Fritch and M. Maniatis. 1989. Molecular cloning: A laboratory manual. $2^{\text {nd }}$ Edition. Cold Spring Harbar Lab Press, New York.

Shivakumar, B.M. 1997. Molecular characterization of Indian cattle breeds using RAPD marker. MVSC Thesis. IVRI, Izatnagar, Bareilly, India.

Stepniak, E., M.M. Zagalska and M. Cwitonsk. 2002. Use of RAPD technique in evolution studies of four species in the family Canidae. J. Appl. Genet. 43(4):489-499. 